\title{
EVALUATION OF POWER FACTOR UNDER LINEAR AND NONLINEAR LOAD CONDITIONS
}

\author{
Mohammed A. Abdulsada \\ mohammed mx20@yahoo.com \\ Furat A. Abbas \\ Fathi R. Abusief \\ furat74@yahoo.com \\ fabusief@yahoo.com \\ Electrical Engineering Department, Faculty of Engineering, \\ Omar Al-Mukhtar University, Tobruk, LIBYA
}

\begin{abstract}
The aim of this paper is to evaluate the power factor of the linear and nonlinear load. The effect of poor power factor on the supply circuit is discussed. The general definitions and equations of power relation are presented. The power factor under linear load condition is derived and discussed. Under nonlinear load, the total power factor is made of two components: displacement factor and distortion factor. The distortion factor associated with the harmonics present. The power factor in terms of Fourier series coefficients is evaluated. The power factor correction (PFC) techniques are discussed for linear and nonlinear load. . As case study, the power factor of three linear different loads is calculated. A 3-phase , 6-pulse, AC/DC converter is taken as an example of nonlinear load and the total power factor when feeding R-load and R-L load is evaluated.

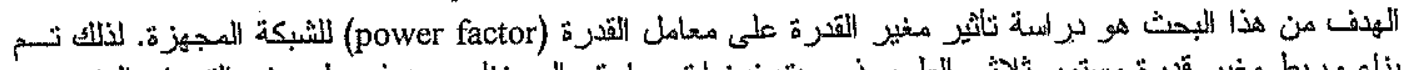

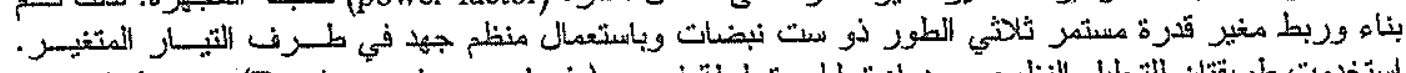

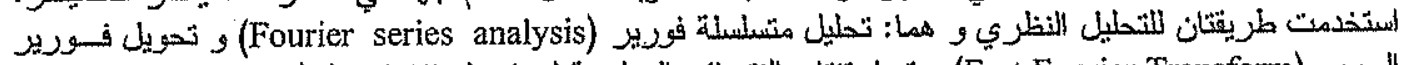

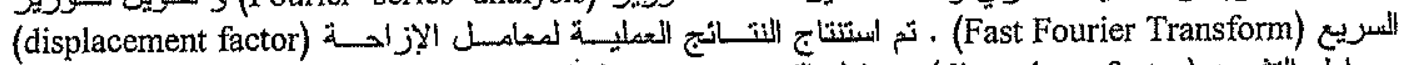

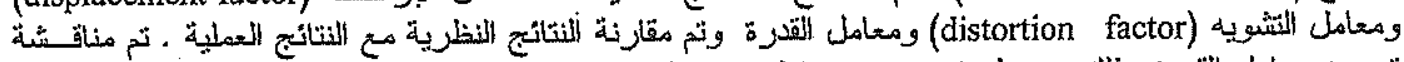

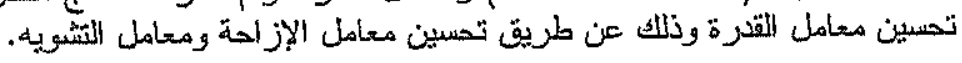

Keywords: Power factor, displacement factor, distortion factor, linear \& nonlinear loads, AC/DC converter, and power factor correction.

\section{INTRODUCTION}

The electrical energy is almost exclusively generated, transmitted and distributed in the form of alternating current. Therefore, the question of power factor immediately comes into picture. The A.C. motors, $\mathrm{AC} / \mathrm{DC}$ converters, arc lamps, arc furnaces, induction heaters etc... are the main causes of low power factor. The poor power factor results in the following disadvantages [1,2]:

(i) Large KVA rating of transformers.

(ii) Large copper losses and greater ac conductor sizes.

(iii) Lower rated power $(\mathrm{kW})$ of the converters.

The power factor of a circuit is universally defined as "the ratio of the active or average power, in watts, to the apparent power or product RMS volt time's RMS amperes at the terminals". The above definition is based on the assumption that loads on the system have liner voltage-current characteristics (i.e. linear load) and that harmonic distortion of the current and voltage is not significant. With these assumptions, the power factor is equal to the displacement factor.

Harmonic distortion in the current and voltage caused by nonlinear loads, such as $\mathrm{AC} / \mathrm{DC}$ converters, changes the way power factor must be defined [3]. In this case, the total power factor is defined as "the ratio of the total power input, in watt, to the total volt-amperes in the circuit", the total power factor made up of two components; displacement and distortion factors. The displacement factor is "the ratio of the active power of the fundamental wave, in watt, to the apparent power, in volt-amperes". This is the power factor that is measured by metering. The distortion factor is that part associated with the presence of harmonic current and voltage.

The association of total power with displacement and distortion factors is important because it highlights the factors which determine the overall power factor of a system. It indicates that different criteria may be adopted in dealing with the problem of power factor correction (PFC) methods. If the power factor is mainly associated with displacement factor, a high power factor may be achieved by the use of energy storage methods of compensation (reactive power compensation). On other hand, if power factor is mainly associated with distortion factor then methods of harmonics elimination must be used to enhance the $\mathrm{AC}$ line currents and some time it is required both of them [4].

In liner loads, such as lightening and AC motors, the PFC through the application of capacitor banks is well known and widely practiced at all voltage level systems. The capacitor banks are connected in 
parallel with the equipment operating at lagging power factor. It draws a leading current and partly or completely neutralizes the lagging reactive power.

The power factor correction (PFC) in nonlinear loads can be made if the distortion factor and displacement factor are improved. The distortion factor is increased by reducing the harmonic currents from entering the main system by providing a shunt filter of low impedance to the harmonic frequencies. These shunt filters also supply all or part of the reactive power consumed by the converter, the remainder being supplied by shunt capacitor banks. In the presence of harmonics, these capacitors can cause parallel or series resonance with the system inductance and the result may be the flow of excessive amount of harmonic current and appearance of harmonic over voltage which may lead to excessive capacitor fuses operation and capacitor bank failure. Another problem is the over voltage at the consumer bus created by switching of the capacitors, which can damage sensitive electronic equipment [5].

The objective of this paper is to analyze the total power factor under linear and nonlinear load condition. The power factor correction (PFC) techniques are discussed. The power factor of three different linear loads is calculated. A 3-phase, 6pulse, AC/DC converter is taken as a nonlinear load and the total power factor in this case is evaluated.

\section{GENERAL DEFINITION OF POWER RELATIONS}

\subsection{Active Input Power (P)}

The active input power entering the network is given by

$$
P=\frac{1}{2 \pi} \int_{0}^{2 \pi} e(\omega t) i(\omega t) d \omega t
$$

Where $e(\omega t)$ and $i(\omega t)$ are the instantaneous supply voltage.

\subsection{Reactive Power (Q)}

The reactive power is that components of apparent power created by combination of $90^{\circ}$ out of phase current component with corresponding voltage component.

\subsection{Apparent Power or Volt-Amperes (S)}

The apparent power is the magnitude of product of the supply voltage and current, both are in root mean square (RMS) values, that is:

$S=\mathrm{E} . \hat{\mathrm{I}}$

\subsection{Input Displacement Angle and Displacement \\ Factor:}

The angle between the fundamental supply voltage and the fundamental component of the supply line current is known as displacement angle $\left(\phi_{1}\right)$. The displacement factor can be defined as the ratio of the active power to the fundamental apparent power and is given by the following equation:

$\operatorname{Cos} \varphi_{1}=\frac{P}{E_{1} I_{L}}$

\subsection{Distortion Factor $(\cos \Delta)$ :}

The distortion factor is defined as the ratio of the RMS amplitude of the fundamental component of the input supply line current to its total RMS amplitude, that is:

$\cos \delta=\frac{I_{1}}{I_{L}}$

\subsection{Total Power Factor (PF):}

The total power factor is defined as the ratio of the total active power input, in watt, to the total apparent power in the circuit, that is:

$$
P F=\frac{\text { Total active power input }(W)}{\text { Total apparent power input }(V A)}
$$

\section{POWER FACTOR UNDER LINEAR LOAD CONDITION}

The linear load is that load draw a sinusoidal current wave when supplied by a sinusoidal supply voltage. Only the fundamental component of the supply voltage and line current are exist and the active input power could be described as:

$$
\begin{aligned}
P & =\frac{1}{2 \pi} \int_{0}^{2 \pi} e(\omega t) \cdot i(\omega t) d \omega t \\
& =\frac{1}{2 \pi} \int_{0}^{2 \pi} \sqrt{2} E_{n} \sin (\omega t) \cdot \sqrt{2} I_{1} \sin \left(\omega t+\varphi_{1}\right) d \omega t
\end{aligned}
$$

Which leads to:

$$
\mathrm{P}=\mathrm{E}_{\mathrm{n}} \cdot \mathrm{I}_{1} \cdot \cos \varphi_{1}
$$

The reactive and apparent power will be:

$$
\begin{aligned}
& Q=E_{n} I_{1} \cdot \sin \phi=S_{1} \cdot \sin \phi \\
& \mathrm{S}=\mathrm{S}_{1}=\mathrm{E}_{\mathrm{n}} \cdot \hat{I}_{1}
\end{aligned}
$$

From the above equations the total power factor is given by:

$$
P \dot{F}=\frac{P}{S_{1}}=\cos \phi_{1} \text {. }
$$

From Eq. (9), it can be deduced that in such loads the total power factor is equal to the displacement factor.

\section{POWER FACTOR UNDER NONLINEAR LOAD CONDITION}

In the case of nonlinear load, the current drawn from the main supply voltage is non-sinusoidal (distorted) and can be represented by Fourier summation of the fundamental and harmonics components [6]:

$$
\begin{aligned}
I(\omega t)= & \sqrt{2} I_{1} \sin \left(\omega t+\phi_{1}\right) \\
& +\sum_{h=2}^{\infty} \sqrt{2} I_{h} \sin \left(h \omega t+\phi_{h}\right)
\end{aligned}
$$


The active power drawn from the supply system due to each harmonic component of current is given by:

$P_{h}=\frac{1}{2 \pi} \int_{0}^{2 \pi} \sqrt{2} E_{n} \sin (\omega t) \cdot \sqrt{2} I_{h} \sin \left(h \omega t+\phi_{h}\right) d \omega t=0$

Equation (11) means the active power transferred only by combination of voltage and current components of the same frequency which is given in Eq. (6).The total RMS line current and total apparent power is given by:

$$
\begin{aligned}
& I_{L}=\sqrt{I_{1}^{2}+\sum_{h=2}^{\infty} I_{h}^{2}}=\sqrt{\sum_{h=1}^{\infty} I_{h}^{2}} \\
& S=E_{n} \cdot I_{L}=E_{n} \cdot \sqrt{\sum_{h=1}^{\infty} I_{h}^{2}}
\end{aligned}
$$

The total power factor will be:

$$
P F=\frac{I}{\sqrt{\sum_{h=1}^{\infty} I_{h}^{2}}} \cdot \cos \phi_{1}=\frac{I_{1}}{l_{L}} \cdot \cos \phi_{1}
$$

Thus, the power factor of nonlinear load is made up of product of two components: displacement factor $\left(\cos \phi_{1}\right)$ and the distortion factor $\left(I_{1} / I_{L}\right)$ which associated with the harmonics present.

Since, the current $I_{L}$ is greater than $I_{l}$ due to the presence of current harmonics components, the distortion factor is less than unity and hence the power factor is less than unity even in those cases where $\left(\cos \phi_{1}\right)$ has it is maximum value of unity.

In the case of non-sinusoidal current, i.e. presence of harmonics, which is generally the case in converters it is necessary to define a further analytical component know as distortion power or distortion volt-amperes (D) which associated with the harmonic currents. The power relations can be represented in a phasor diagram as shown in Fig.1.

The total apparent power in this case is given by [7]:

$S^{2}=P^{2}+Q^{2}+D^{2}$

Since the only voltage component is the sinusoidal supply voltage by neglecting the supply impedance, the only relevant current component is the quadrate component of fundamental current, this lead directly to Eq. (7) for reactive power that is complement of Eq. (6) for active power. The distortion power will be:

$$
D^{2}=E_{n}^{2}\left(\sum_{h=2}^{\infty} I_{h}^{2}\right)
$$

\section{POWER FACTOR IN TERMS OF FOURIER SERIES COEFFICIENTS}

The total power factor can be calculated by using the Fourier series analysis. For a periodic non-sinusoidal current $i(\omega t)$ of a periodicity $2 \pi$ radians the Fourier coefficient al and $b_{1}$ for the peak value of the fundamental line current are given as the following [8]:

$$
\begin{aligned}
& a_{1}=\frac{1}{\pi} \int_{0}^{2 \pi} i(\omega t) \cdot \cos \omega t d \omega t \\
& b_{1}=\frac{1}{\pi} \int_{0}^{2 \pi} i(\omega t) \cdot \sin \omega t d \omega t
\end{aligned}
$$

The RMS value of fundamental line current $\left(I_{1}\right)$ is given by:

$$
I_{1}=\sqrt{\left(a_{1}^{2}+b_{1}^{2}\right) / 2}
$$

The displacement factor can be calculated as follows:

$$
\cos \phi_{1}=\cos \left(\tan ^{-1} \frac{a_{1}}{b_{1}}\right)=\frac{b_{1}}{\sqrt{a_{1}^{2}+b_{1}^{2}}}
$$

The distortion factor is given by:

$$
\cos \delta=\frac{\sqrt{a_{1}^{2}+b_{1}^{2}}}{\sqrt{2} I_{L}}
$$

The total power factor is found to be:

$$
P F=\frac{b_{1}}{\sqrt{2} I_{l}}
$$

\section{POWER FACTOR CORRECTION TECHNIQUES}

The power factor under linear loads can be corrected by connecting the static power capacitors (capacitor bank) in parallel with the loads. The capacitor banks are an inexpensive source of reactive power. They have the advantages of small losses, low initial cost, little maintenance, easy installation and capability to operate under ordinary atmosphere conditions. However, they have drawbacks of short service life ( 8 to 10 years), getting damaged on over-voltage and uneconomical repair. In case of 3-phase loads capacitor bank can be connected either in star or delta. Depending on the total VARS requirements, a number of capacitors are used which can be switched into or switched out of the system individually [2].

The leading KVAR supplied by the capacitor bank is given by:

$\mathrm{KVAR}=\mathrm{P}_{\mathrm{L}}\left(\tan \varphi_{1}-\tan \varphi_{2}\right)$

Where $\varphi_{2}$ is the corrected displacement angle.

If the losses are neglected, then KVAR per phase will be equals the KV per phase and the value of capacitor per phase $(C)$ can be found as follows:

$C=\frac{K V A R \text { perphase }}{2 . \pi \cdot f \cdot E_{n}^{2} \cdot I_{L}}$

Another method of PFC in linear loads is by using the synchronous machines which can be made to operate with either a leading or lagging power factor by controlling the field excitation. A synchronous machine is referred to as synchronous condenser 
when it is dedicated slowly to reactive power compensation. The synchronous condenser is more expensive and much greater losses than capacitor banks; however it injects or absorbs reactive power smoothly.

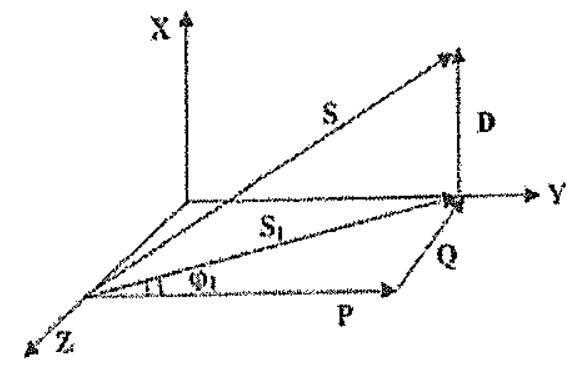

Fig.(1) Power phasor diagram of a nonlinear load

For the purpose of PFC in case of nonlinear loads, the two factors of interest are the distortion and displacement factors. The aim of PFC is to make the product of these two factors to be unity $[4,9]$.

The displacement factor can be improved by furnishing reactive power (KVARs) by external source at the supply terminals, which supply and compensate the lagging reactive power consumed by the converters. The main sources of reactive power are capacitor banks and synchronous condensers as explained above.

The distortion factor is always less than unity. Improvement in the value of distortion factor can be done by reducing the level of current harmonics using filters, higher pulse number of converter, line current waveform modification, and other methods of harmonics suppression [1].

\section{CASE STUDY OF LINEAR AND NONLINEAR LOADS}

\subsection{Linear Load}

In a purely resistive circuit, the current $\left(I_{R}\right)$ and the applied voltage (e) are in phase. The total power factor (i. e. displacement factor) is given by:

$$
\begin{aligned}
& P F=\cos \varphi_{1}=\frac{R}{Z}=\frac{R}{R}=1 \quad \text { (i.e. unity) } \\
& P F=\cos \varphi_{1}=\frac{R}{Z}=\frac{R}{\sqrt{R^{2}+X_{L}^{2}}} \quad \text { lagging }
\end{aligned}
$$

In a circuit containing inductance in series with resistance ( $R-L$ Load), the current $\left(I_{L}\right)$ lags the applied voltage by an angle $\left(\phi_{1}\right)$. The power factor for such circuit is given by:

$$
P F=\cos \varphi_{1}=\frac{R}{Z}=\frac{R}{\sqrt{R^{2}+X_{L}^{2}}} \text { lagging }
$$

\subsection{Nonlinear Load}

Diodes and circuits bridge converters are considered as the most important and practical in the power electronics universe. The 3-phase, 6-pulse bridge converters are widely employed in the industry; therefore it is taken as a case study of nonlinear load.

If the three-phase bridge converter feeding a resistive load ( $R$ load), there are two distinct modes of operation for the converter depending upon the firing angle.

In the range of firing angle $0 \leq \alpha \leq 60^{\circ}$ there is no possibility for the load voltage to become negative. Therefore, a load current does not become zero. In this range of firing angle, conduction is continues.

In the range of firing angle $60^{\circ} \leq \alpha \leq 120^{\circ}$, load voltage tends to become negative when line voltage passes through zero crossing. The resistance load does not allow this and the voltage across load is maintained at zero and current is also zero. Conduction of converter in this range of firing angles is discontinuous.

Fig.2 shows the waveforms of dc load voltage (heavy line), secondary and supply line currents at no firing angle $(\alpha=0)$ and with resistive load.

Both the load current and the secondary line currents are made up of parts of sinusoids, the primary phase current waveforms in the star connected winding are the same as those in the corresponding secondary star connected winding divided by the turn's ratio $(\mathrm{T})$.
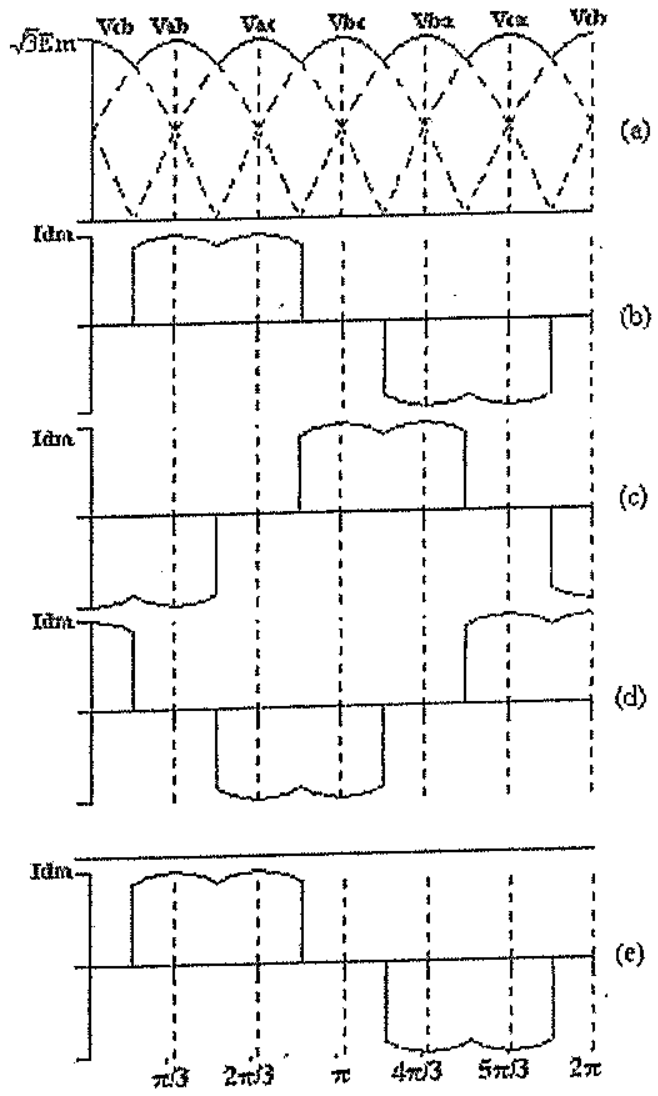

Fig.2 Waveforms of (a) dc load voltage (heavy line), (b,c,d) secondary line currents (e) supply line currents 
The displacement, distortion and total power factor at the supply side can be evaluated in terms of Fourier coefficients. The displacement factor can be found to be:

$$
\cos \phi=\left\{\begin{array}{ll}
\frac{2 \pi+3 \sqrt{3} \cos 2 \alpha}{\left[27+4 \pi^{2}+12 \sqrt{3} \pi \cos 2 \alpha\right]^{7 / 2}} & 0 \leq \alpha \leq 60^{\circ} \\
\frac{4 \pi-6 \alpha-3 \sin (2 \alpha-\pi / 3)}{\left[9\left(1+\cos \left(2 \alpha-60^{\circ}\right)^{2}+(4 \pi-6 \alpha-3 \sin (2 \alpha-\pi / 3))^{2}\right]^{\frac{2}{2}}\right.} & 60^{\circ} \leq \alpha \leq 120^{\circ}
\end{array}\right\}
$$

Combination of Eq.(6), the Fourier coefficients, and Eq.(21), results in the following expression for distortion factor:

$$
\cos \delta=\left\{\begin{array}{ll}
{\left[\frac{27+4 \pi^{2}+12 \sqrt{3} \pi \cos 2 \alpha}{4 \pi(2 \pi+3 \sqrt{3} \cos 2 \alpha)}\right]^{1 / 2}} & 0^{\circ} \leq \alpha \leq 60^{\circ} \\
{\left[\frac{9[1+\cos (2 \alpha-\pi / 3)]^{2}+\left[4 \pi-6 \alpha-3 \sin (2 \alpha-\pi / 3]^{2}\right.}{4 \pi[4 \pi-6 \alpha-3 \sin (2 \alpha-\pi / 3]}\right]^{1 / 2}} & 60^{\circ} \leq \alpha \leq 120^{\circ}
\end{array}\right\}
$$

The supply total power factor is obtained using Eq. (22), or can be expressed as the product of Eq. (28) and (29) is given by:

$$
\text { P.F }=\left\{\begin{array}{ll}
{\left[\frac{2 \pi+3 \sqrt{3} \cos 2 \alpha}{4 \pi}\right]^{1 / 2}} & 0^{\circ} \leq \alpha \leq 60^{\circ} \\
{\left[\frac{4 \pi-6 \alpha-3 \sin (2 \alpha-\pi / 3)}{4 \pi}\right]^{1 / 2}} & 60^{\circ} \leq \alpha \leq 120^{\circ}
\end{array}\right\}
$$

Fig. 3 shows the variation of displacement, distortion, and total power factors with firing angle.

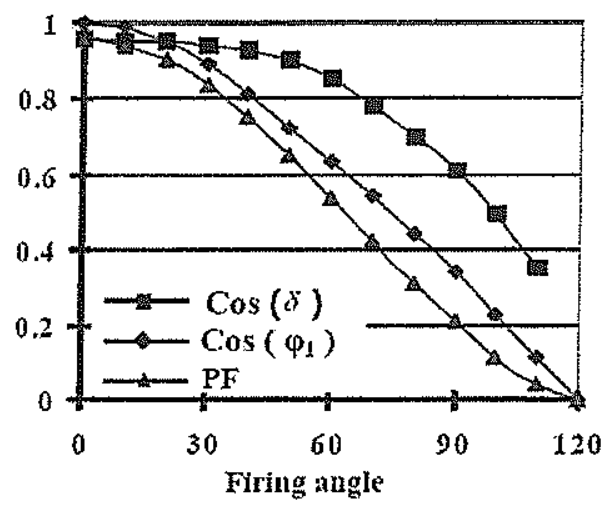

Fig.(3) Distortion factor, displacement factor and total power factor vs. firing angle for resistive load.

When the 3-phase bridge converter feeding highly inductive load (R-L load), the operation of the converter for $\alpha>90^{\circ}$ is not possible. The converter operated as a rectifier only. The control angle range is less than that of a converter feeding only resistive load .The voltage variation is from positive maximum to zero for $\alpha$ varying from $0^{\circ}$ to $90^{\circ}$. If the $\mathrm{dc}$ inductance has a large value then the load current approaches the waveform of pure dc. The supply line current is free from ripple and waveform will be as shown in Fig.4 for firing angles $0^{\circ}$ and $60^{\circ}$.
The displacement factor can be given as:

$$
\cos \left(\phi_{1}\right)=\frac{b_{1}}{\left[a_{1}^{2}+b_{1}^{2}\right]^{3 / 2}}=\cos (\alpha)
$$

Therefore, the displacement angle $\left(\phi_{1}\right)$ between the supply voltage and fundamental component of supply current is equal to the firing angle $(\alpha)$. The displacement factor is unity at $\alpha=0$. The distortion factor for the supply line current waveform of Fig. 4 is constant because the waveform retains the same form for all values of $\alpha$ and has value of $(\pi / 3)$, that is:

$\cos (\delta)=\frac{3}{\pi}=0.955$

The variation of displacement, distortion and total power factor with the firing angle are shown in Fig. 5.

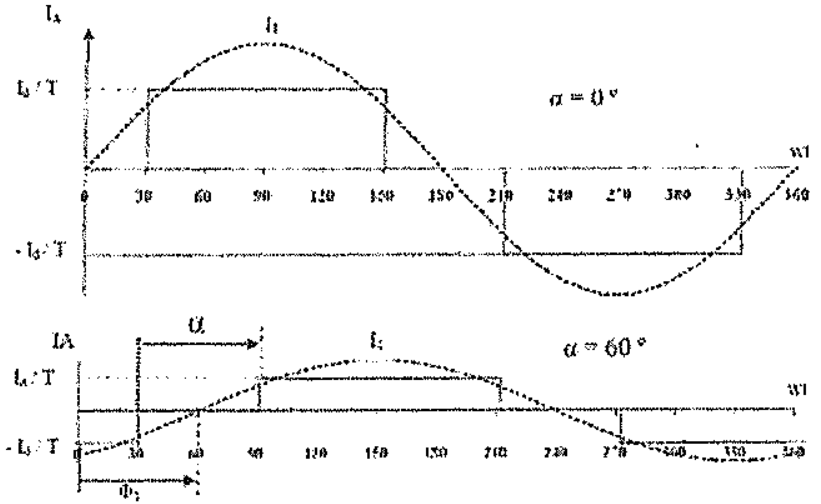

Fig. (4) Supply line current and fundamental waveforms for $\alpha=0$ and $\alpha=60^{\circ}$ with highly inductive load.

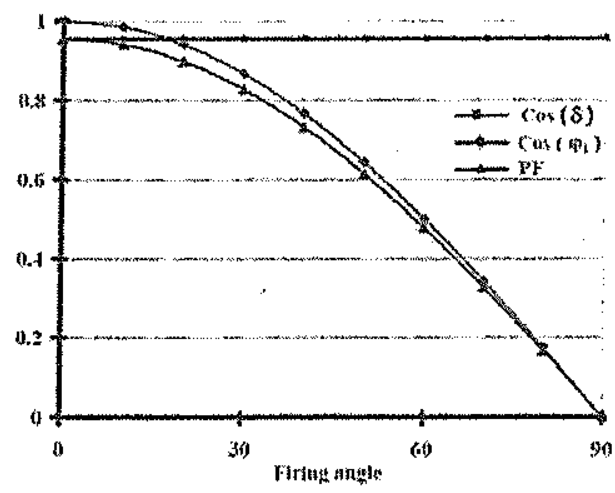

Fig.(5) Distortion factor, Displacement factor and total power factor versus Firing angle for highly inductive load

\section{CONCLUSION}

In this paper, the total power factor under linear and nonlinear load condition is presented. The total power factor expressed as a product of distortion factor and displacement factor. In linear loads the distortion factor is unity and the total power factor equals to the displacement factor. In nonlinear loads, the distortion effect of the higher harmonic components of the supply line current is expressed by 
the distortion factor. The displacement factor, distortion factor total power factor of a 3-phase, 6pulse, AC/DC converter feeding R-load \&R-L load are derived. The total power factor is a function of firing angle $\alpha$, as $\alpha$ increase the power factor is decreased. In case of converter feeding high inductive load, it is found that the distortion factor is a constant and has a value of $3 / \pi$. The displacement factor is responsible of low power factor and improvement of its value is recommended. The displacement factor is responsible of low power factor and improvement of its value is recommended.. The disadvantages of low power factor leads to the conclusion that it is an objectionable feature in the supply system. Although power factor improvement involves extra annual expenditure on account of PFC equipment, yet improvement of power factor to a proper value results in the net annual saves for both the main supply and the consumer.

\section{REFERENCES}

[1] J. Arrillaga, et al, "Power System Harmonic Analysis", 1997, Book, and London: Wiley.

[2] V. K. Mehta, "Principle of Power System", 1998, Book, S. Chand and Company Itd., India.

[3] IEEE standard 519 - 1992, "IEEE Recommended Practices and Requirements for Harmonic Control in Electrical Power Systems".

[4] H. Mao, etal, "Review of High Performance Three Phase Power Factor Correction Circuits", IEEE Trans. On Industry electronics, Vol.44, No. 4, Aug. 1997, PP 437-445.

[5] R. D. Henderson and P. J. Rose, "Harmonics: the Effect on Power Quality and Transformer", IEEE Trans. On Industry Applications, Vol.30, No. 3, May/June 1994, PP 528-532.

[6] J. S. Subjak and J. S. Mequilken, "Harmonics Causes, Effects, Measurements and Analysis: An Update", IEEE Trans. On Industry Applications, Vol.26, No. 6, Nov/Dec. 1990, PP 1034-1042.

[7] Task Force on Harmonics Modeling and Simulation, "Modeling and Simulation of the
Propagation of Harmonics in Electric Power Networks", IEEE Trans. on Power delivery, Vol.11, No. 1, Jan. 1996, PP 452-461.

[8] W. Shepherd and P. Zand, "Energy Flow and Power Factor in No Sinusoidal Circuits", 1979, Book, Cambridge university press.

[9] Y. Jang and M. Jovanovic, "A Comparative Study Of Single Switch, Three Phase, High Power Factor Rectifier", IEEE APEC'98 Conference record, Part 2, PP 1093-1099.

\section{LIST OF SYMBOLS}

$\begin{array}{ll}\alpha & \text { Firing angle. } \\ \varphi_{1} & \text { Displacement angle. } \\ \varphi_{2} & \text { Corrected displacement angle. } \\ \phi_{\mathrm{h}} & \text { Harmonic phase angle between the } h^{\prime} \text { th line } \\ & \text { and fundamental phase voltage. }\end{array}$

$\operatorname{Cos} \varphi_{1}$ Displacement factor.

$\operatorname{Cos} \delta$ Distortion factor.

D Distortion power.

$E_{d} \quad$ Average direct voltage.

$E_{n} \quad A C$ supply line-to-neutral voltage (RMS).

$E_{m} \quad$ Maximum value of secondary phase voltage.

h Order of harmonic.

$\mathrm{i}(\omega \mathrm{t})$ Instantaneous supply current

$I_{d} \quad$ Average value of $d c$ load current.

$\mathrm{I}_{\mathrm{dm}} \quad$ Crest value of dc load current.

$I_{1} \quad$ Fundamental component of $I_{L}$.

$\mathrm{I}_{10} \quad$ Fundamental component of $\mathrm{I}_{\mathrm{L}}$, assuming zero phase control.

$\mathrm{I}_{\mathrm{L}} \quad$ Supply line current (RMS).

$\mathrm{K}$ Integer no. , $1,2,3, \ldots$

$\mathrm{P} \quad$ Active input power.

PF Total power factor.

$R \quad$ Resistance of dc load.

S Total apparent power.

$\mathrm{S}_{\mathrm{I}} \quad$ Fundamental component of apparent power.

$T$ Transformer turns ratio.

$V_{A}, V_{B}, V_{C} \quad$ Instantaneous line-to-neutral 3-phase supply voltages.

$V_{A B}, V_{B C}, V_{A C}$ Instantaneous line-to-line 3-phase supply voltages. 